\title{
Predicting lymph node status in early gastric cancer
}

\author{
Robert Michael Kwee ${ }^{1}$ and Thomas Christian KweE ${ }^{2}$ \\ ${ }^{1}$ Department of Radiology, University Medical Center Maastricht, P. Debyelaan 25, 6202 AZ, Maastricht, The Netherlands \\ ${ }^{2}$ Department of Radiology, University Medical Center Utrecht, Utrecht, The Netherlands
}

\begin{abstract}
Accurate prediction of lymph node (LN) status is of crucial importance for appropriate treatment planning in patients with early gastric cancer (EGC). However, there is no definitive consensus yet on which patient and/or tumor characteristics are associated with LN metastasis. A systematic search for studies investigating the relationship between patient and/or tumor characteristics and LN metastasis in EGC was performed in PubMed/MEDLINE. Patient and/or tumor characteristics associated with $L N$ metastasis were identified by meta-analyzing results of individual studies. Forty-five studies were included. Variables significantly associated with LN metastasis in gastric cancer limited to the mucosa were: age younger than 57 years, tumor location in the middle part of the stomach, larger tumor size, macroscopically depressed tumor type, tumor ulcerations, undifferentiated tumors, diffuse tumor type according to the Lauren classification, lymphatic tumor invasion, tumors with a proliferating cell nuclear antigen (PCNA) labeling index of more than $25 \%$, and matrix metalloproteinase-9-positive tumors. Variables significantly associated with LN metastasis in gastric cancer limited to the submucosa were: female sex, tumor location in the lower part of the stomach, larger tumor size, undifferentiated tumors, increasing depth of submucosal invasion, lymphatic tumor invasion, vascular tumor invasion, increased submucosal vascularity, tumors with a PCNA labeling index of more than $\mathbf{2 5} \%$, tumors with a gastric mucin phenotype, and vascular endothelial growth factor-C-positive tumors. We identified several variables associated with LN metastasis in EGC. These variables should be included in future research, in order to assess which of these variables remain as significant predictors of LN metastasis.
\end{abstract}

Key words Early gastric cancer - Lymph node metastasis · Prediction $\cdot$ Systematic review $\cdot$ Meta-analysis

\section{Introduction}

Early gastric cancer (EGC) is defined as gastric cancer confined to the mucosa or submucosa, regardless of the presence or absence of lymph node (LN) metastasis [1]. In the Eastern hemisphere, up to $70 \%$ of all gastric cancers are diagnosed as EGCs (due to mass population screening), whereas in the Western hemisphere, the rate of gastric cancers identified as EGCs accounts for only about $15 \%$ [2]. EGC shows a favorable prognosis compared to advanced gastric cancer, with 5-year cancer-specific survival rates exceeding 95\% [3]. Accurate prediction of LN status is of crucial importance for appropriate curative treatment planning in EGC; LNnegative patients can be curatively treated with minimally invasive endoscopic mucosal resection (EMR) or endoscopic submucosal dissection (ESD) [3], whereas LN-positive patients should undergo (sub) total gastrectomy with limited or extended LN dissection [4]. Gastrectomy with LN dissection is associated with high morbidity and mortality [4], and postoperative quality of life may be impaired because of weight loss, loss of appetite, and other metabolic and nutritional changes. Therefore, this aggressive surgical approach should be reserved only for EGC patients at high risk of LN metastasis. In an attempt to obtain predictive parameters, multiple studies have identified pathologic characteristics of the primary tumor that are associated with an increased likelihood of LN metastasis. Because established eligibility criteria for endoscopic resection of EGC [5] are debated [3, 6], we undertook a systematic review and meta-analysis to give more insight into those patient and/or tumor characteristics that emerge as associated with LN metastasis in EGC. 


\section{Methods}

\section{Data sources}

A computer-aided search of the PubMed/MEDLINE database was conducted to find English-language studies which reported patient and/or tumor characteristics in relation to LN metastasis in patients with EGC. The following search term was used: "node metastasis" or "node metastases" or "nodal metastasis" or "nodal metastases" or "node involvement" or "nodal involvement" or "metastatic nodes" or "metastatic lymph nodes" or "lymphatic metastasis" or "lymphatic metastases" or "lymphatic involvement" or "lymph node metastatic disease" and ("gastric cancer" or "stomach cancer" or "gastric carcinoma" or "stomach carcinoma"). No beginning date limit was used. The search was updated until 30 October, 2007. To expand the search, bibliographies of articles which finally remained after the selection process were screened for potentially suitable references.

\section{Eligibility criteria}

Original studies which investigated the relationship between patient and/or tumor characteristics and the presence or absence of LN metastasis in patients with histopathologically proven EGC were eligible for inclusion. EGC was defined as a tumor histopathologically confined to the mucosa or submucosa, regardless of disease duration, tumor size, presence of symptoms, presence of metastases, or curability [1]. Because risk of LN metastasis is substantially higher in gastric cancer invading the submucosa (submucosal cancer) than in gastric cancer limited to the mucosa (mucosal cancer), only studies which provided separate data for mucosal and/or submucosal cancer were included. Studies performed in animals, ex vivo studies, review articles, meta-analyses, abstracts, editorials or letters, case reports, studies investigating 15 or fewer patients, tutorials, and guidelines for management were excluded. Studies which only investigated the association between patient and/or tumor characteristics and immunohistochemically detected LN micrometastases were also excluded, because these LNs are regarded as pathologically negative for metastatic disease [7]. Only studies dealing with carcinoma were included, because this is overwhelmingly the most important and most common malignant tumor that occurs in the stomach (range, $90 \%$ to $95 \%$ ) [1]. Studies which investigated only a specific type of gastric cancer (i.e., differentiated or undifferentiated gastric cancer, gastric cancer confined to a specific part of the stomach, or depressed gastric cancer) were excluded. Only studies which provided sufficient data to construct a $2 \times 2$ contingency table to calculate the association of one or more patient and/or tumor characteristics with LN metastasis were included. Among studies that (possibly) included overlapping patient populations, the article with the highest number of patients was selected for further analysis. However, (possibly) overlapping studies comprising a lower number of patients were also included if they investigated other patient and/or tumor characteristics than the ones in the study with the highest number of patients. In such cases, data analysis was performed only for the patient and/or tumor characteristics or type of EGC (i.e., either mucosal or submucosal cancer), which had not been investigated in the study with the highest number of patients.

Titles and abstracts of the retrieved articles were screened by two researchers (R. M. K. and T. C. K.), using the inclusion and exclusion criteria as mentioned above. Articles were rejected if they were clearly ineligible. Full-text versions of all articles that were found to be potentially eligible for inclusion were then evaluated to make a final decision regarding inclusion or exclusion. Discrepancies between the two researchers were solved by consensus.

\section{Data analysis}

For each included study, information was collected concerning the year of publication, country of origin, patient acquisition (consecutive vs nonconsecutive), number of investigated patients, percentage of patients with $\mathrm{LN}$ metastasis, extent of lymphadenectomy, variables in relation to LN metastasis analyzed in this review, blind assessment of variables to LN status, and blind assessment of $\mathrm{LN}$ status to the investigated variables.

Because of the use of various definitions, the variables "macroscopic tumor type", "histological type", "depth of mucosal or submucosal invasion", "vascular tumor invasion", and "lymphatic tumor invasion" were classified into dichotomized groups. Macroscopic tumor type was divided into depressed and nondepressed types. Among studies which applied the Japanese classification of gastric carcinoma [8], type IIc lesions, type III lesions, and mixed lesions in which either a type IIc or a type III component was present were classified as being depressed. Type I, type IIa, type IIb, and mixed lesions in which no type IIc or type III component was present were classified as being nondepressed. Lesions were excluded from analysis if it was unclear whether or not they were composed of a type IIc or type III component. Histological tumor type was divided into: (1) differentiated and undifferentiated type, or (2) defined according to the Lauren classification (which classifies gastric cancer into either intestinal or diffuse type) [9]. Well- and moderately differentiated tubular or papillary adenocarcinomas were classified as being 
differentiated. Poorly differentiated adenocarcinomas and signet-ring cell carcinomas were classified as being undifferentiated. Mucinous adenocarcinomas were classified as either differentiated or undifferentiated, depending upon the other predominant elements (tubular, papillary, poorly differentiated, or signet-ring cell) [8]. Mucinous adenocarcinomas which could not be classified were excluded from analysis. If a study provided sufficient data, analyses for both histological classifications (1 and 2) were made. Depth of tumor invasion in cancer limited to the mucosa was classified as either presence or absence of invasion into the muscularis mucosae. Depth of tumor invasion in cancer limited to the submucosa was classified as follows: (1) submucosal invasion of more or less than $500 \mu \mathrm{m}$, or (2) presence or absence of invasion beyond the upper third or middle third of the submucosa. If a study provided sufficient data, analyses for both classifications of tumor invasion depth (1 and 2) were made. Vascular and lymphatic tumor invasion were both dichotomized into either presence or absence. If a study provided insufficient data, making a dichotomized classification of either macroscopic tumor type, histological type, depth of tumor invasion, vascular tumor invasion, or lymphatic tumor invasion not possible, no analysis of that tumor parameter was made for the study concerned.

The numbers of patients with metastasis-positive and metastasis-negative LNs for each patient and/or tumor characteristic were abstracted from each study. A standard correction of adding 0.5 to all cells of the $2 \times 2$ contingency table was applied if there were no patients in one of the four cells. Study-specific odds ratios with $95 \%$ confidence intervals (CIs) were calculated for positive versus negative LN status for each tumor/patient characteristic under investigation. The common odds ratios across studies were estimated by means of a Mantel-Haenszel fixed-effects model, weighting individual studies by their variance [10]. Point estimates and 95\% CIs for the common odds ratios were reported for characteristics supported by abstracted data from at least two studies. Confidence intervals that did not overlap the referent (1.0) were considered to be statistically significant. Heterogeneity was tested using the Higgins and Thompson test (Higgins et al. [11]), calculating the $\mathrm{I}^{2}$ statistic. This statistic uses the conventional Cochran's Q statistic to calculate the percentage of total variation across studies that can be attributed to interstudy heterogeneity, ranging from 0 (no heterogeneity) to $100 \%$ (all variance due to heterogeneity). In contrast to Cochran's Q, the $\mathrm{I}^{2}$ is less affected by the number of studies included in a meta-analysis [11]. If no or moderate heterogeneity is found $\left(\mathrm{I}^{2} \leq 50 \%\right)$, pooling is justified. Statistical analyses were executed using Meta-DiSc statistical software version 1.4 (Unit of Clinical Biostatistics, Ramón y Cajal Hospital, Madrid, Spain).

\section{Results}

The computer-aided search revealed 6246 articles from PubMed/MEDLINE. After screening titles and abstracts, 147 articles remained for possible inclusion. After reviewing the full article, 102 articles were excluded. Reasons for exclusion were: no separate analysis for mucosal and/or submucosal cancer was made or could be extracted ( $n=71$ ), insufficient data to construct a $2 \times 2$ contingency table to calculate the association of one or more patient and/or tumor characteristics with LN involvement $(n=13)$, no association between any patient and/or tumor characteristic and LN status in either mucosal or submucosal cancer was investigated $(n=10)$, same data used in another article by the same group, comprising a larger number of patients $(n=4)$, and only the association between patient and/or tumor characteristics and immunohistochemically detected LN micrometastases was determined $(n=4)$. Eventually, 45 studies were included in this systematic review [6, 12-55]. Screening the references of these articles did not result in other potentially relevant articles. The characteristics of the included studies are presented in Table 1. No studies meeting the inclusion criteria had a prospective study design. The number of patients per study for mucosal cancer varied between 18 and 3584 (median, 269). The number of patients per study for submucosal cancer varied between 22 and 2625 (median, 170). Twenty-six studies stated that patients were included in a consecutive way. Among these studies, the percentage of $\mathrm{LN}$-positive cases in patients with mucosal cancer varied between 0.0 and $20.3 \%$ (median, $3.2 \%$ ). For submucosal cancer, the percentage of LN-positive patients varied between $10.2 \%$ and $33.3 \%$ (median, $19.2 \%$ ). In total, 18 different patient and/or tumor characteristics were investigated for mucosal cancer. For submucosal cancer, 24 different patient and/or tumor characteristics were investigated.

\section{Mucosal cancer}

In patients with mucosal cancer, there was no significant association between sex, depth of mucosal tumor infiltration, submucosal vascularity, infiltration of dendritic cells, gastritis cystica profunda (GCP)-like glandular proliferation of the tumor, DNA ploidy, mucin phenotype, matrix metalloproteinase (MMP)-2 expression, and erbB-2 expression, and the presence of $\mathrm{LN}$ metastasis.

Variables significantly associated with LN metastasis were: age younger than 57 years, tumor location in the middle part of the stomach, larger tumor size, macroscopically depressed tumor type, tumor ulcerations, undifferentiated tumors, diffuse tumor type according to the Lauren classification [9], lymphatic tumor 


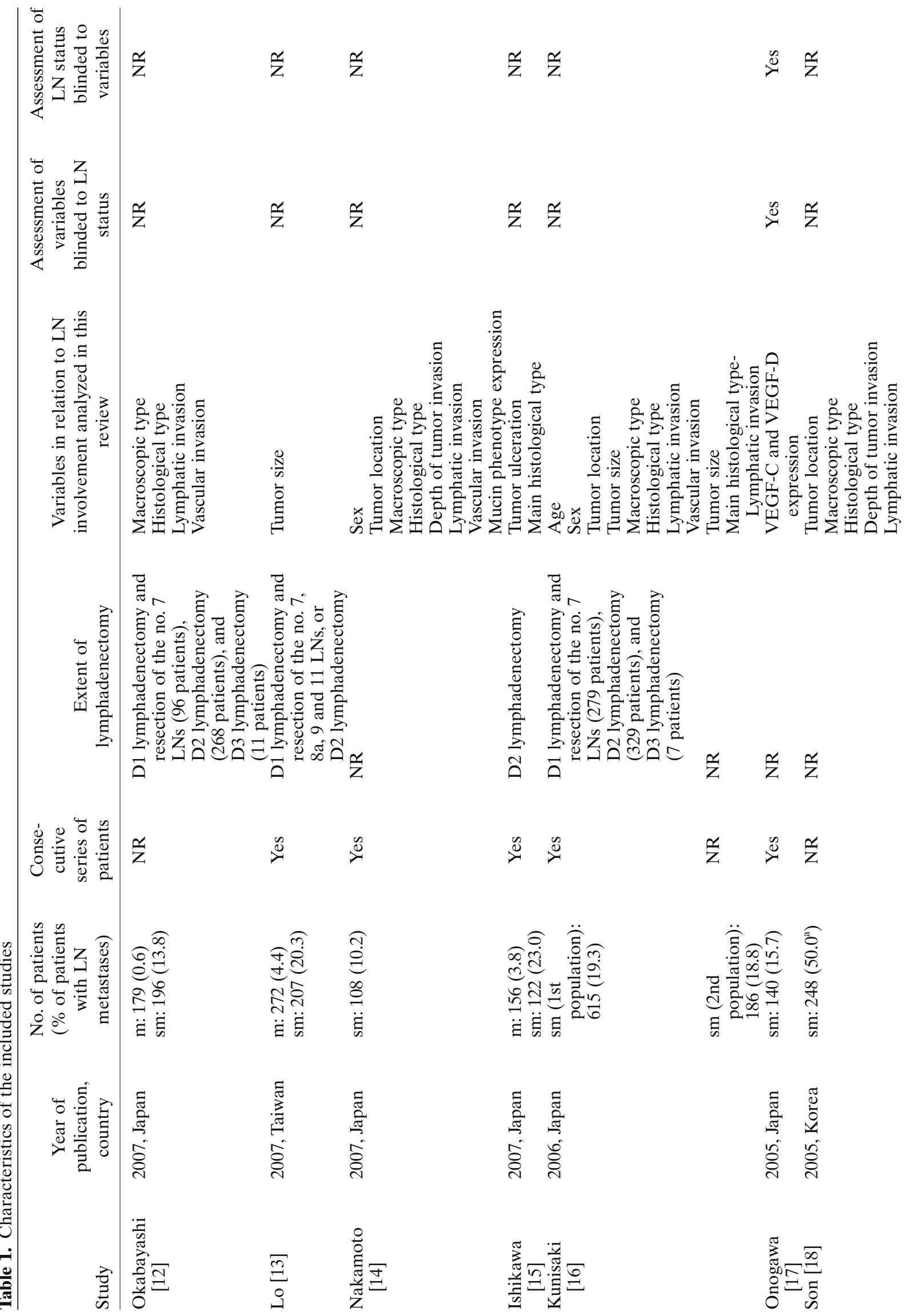




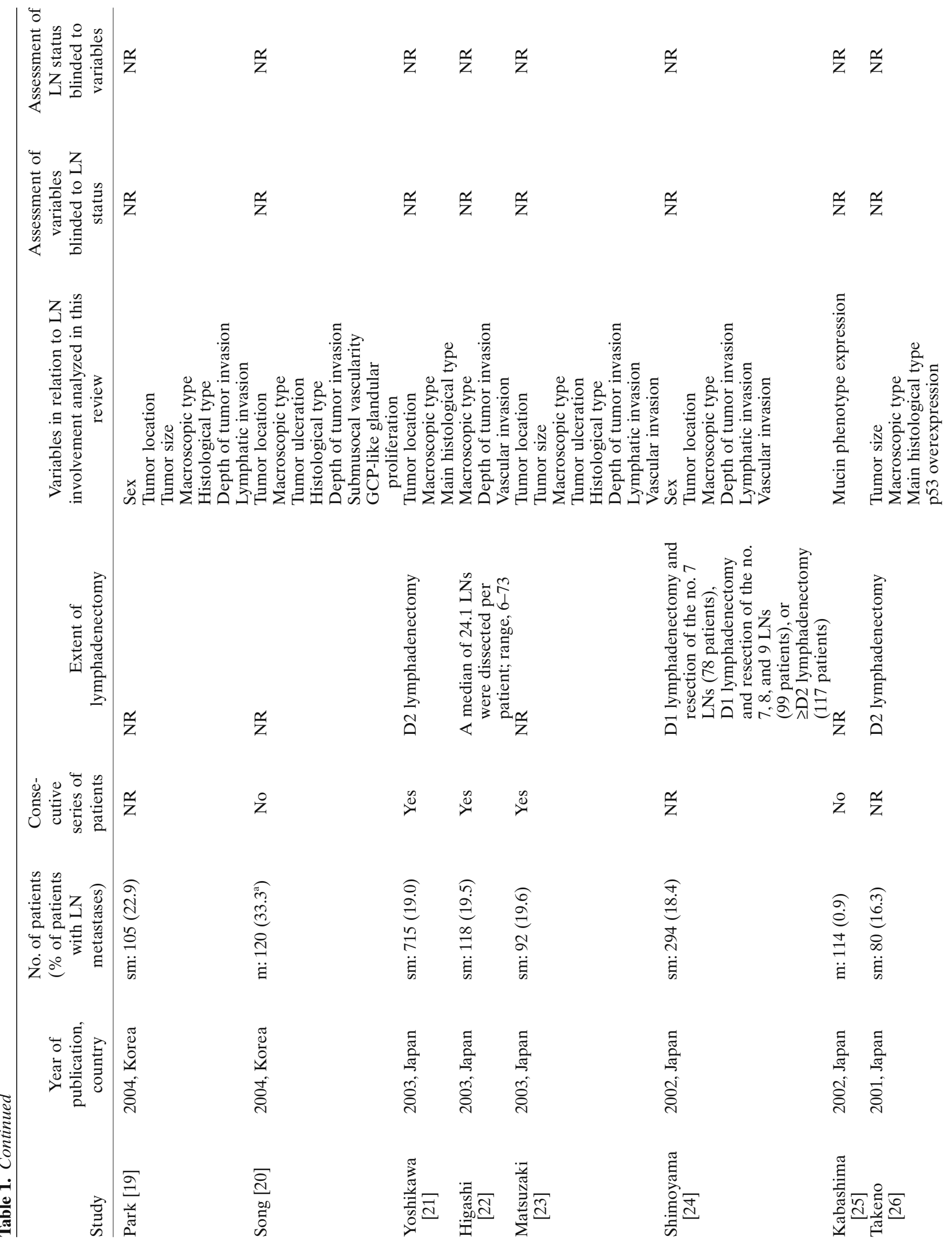




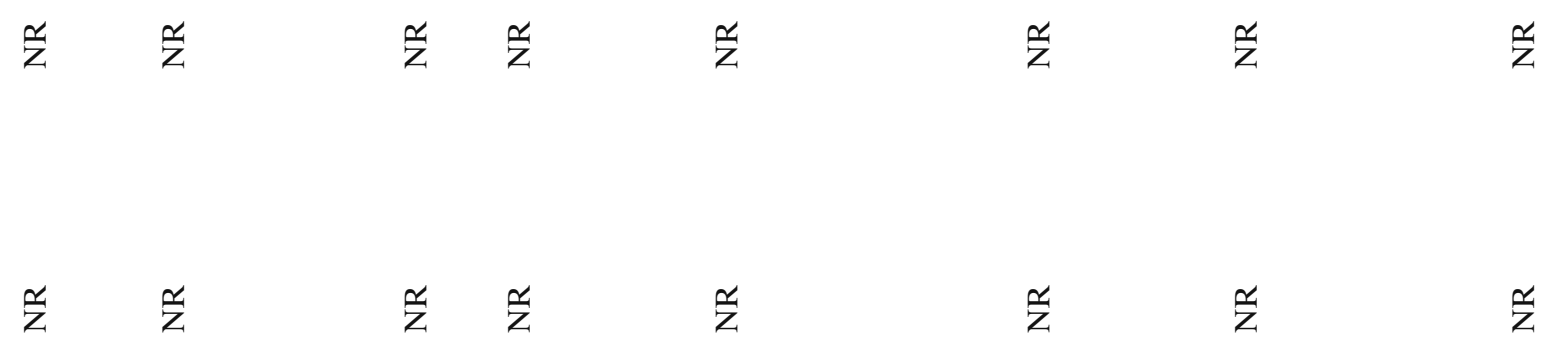
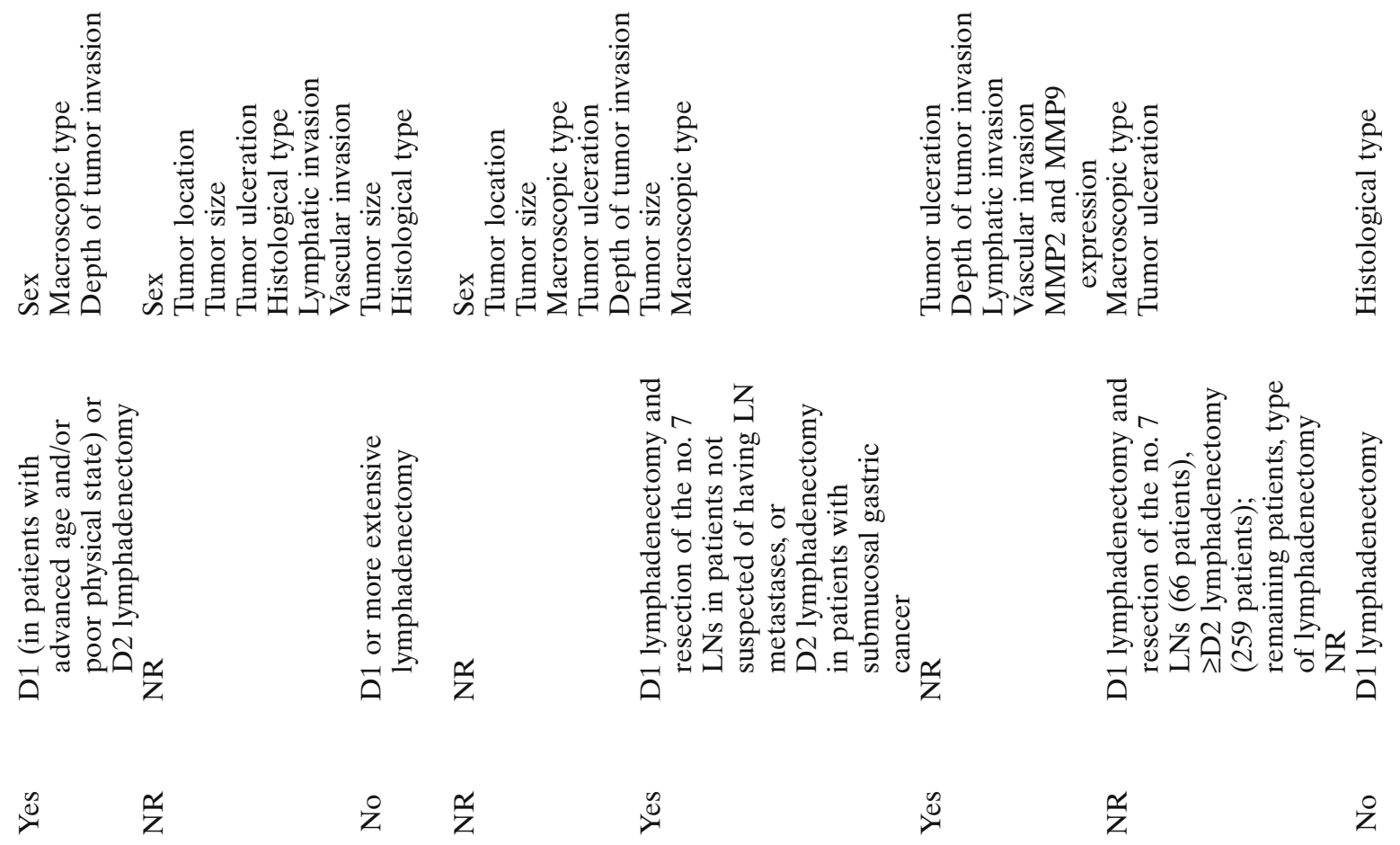

$\stackrel{\infty}{*}$

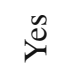

草

₹

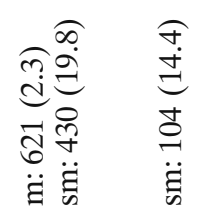

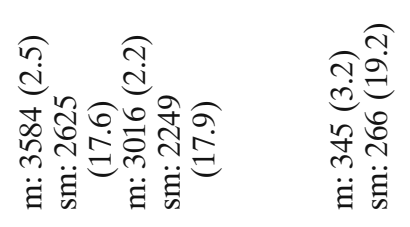

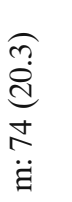

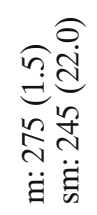

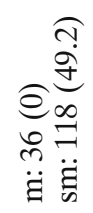

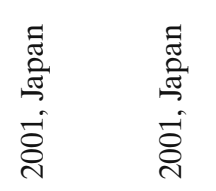

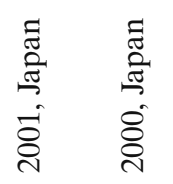

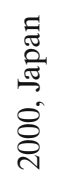

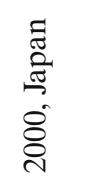

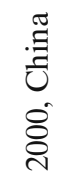

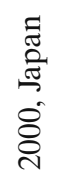

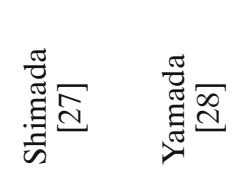

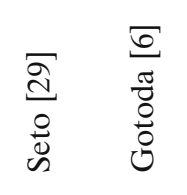

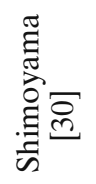

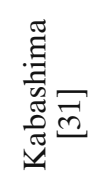

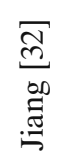




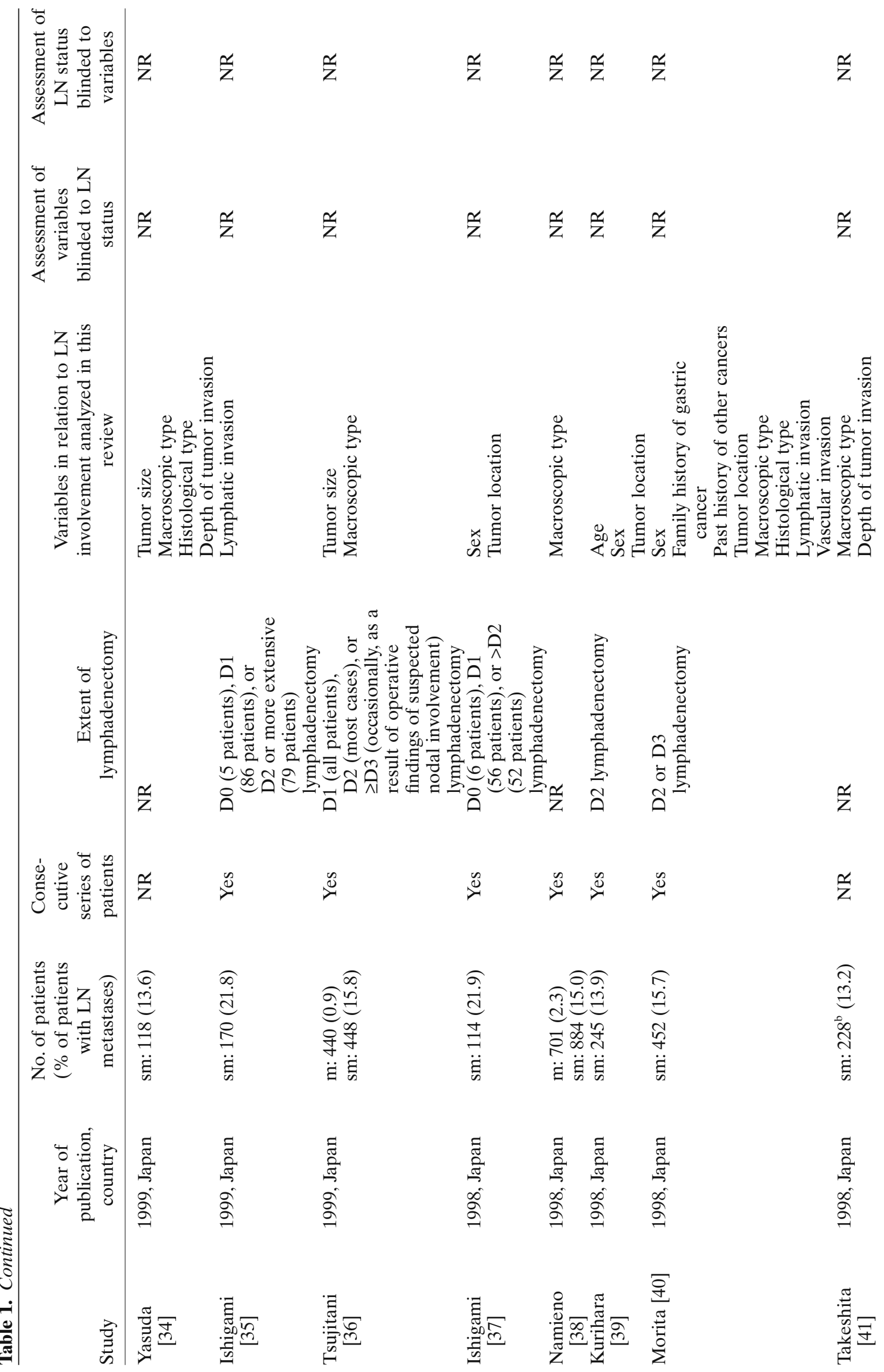




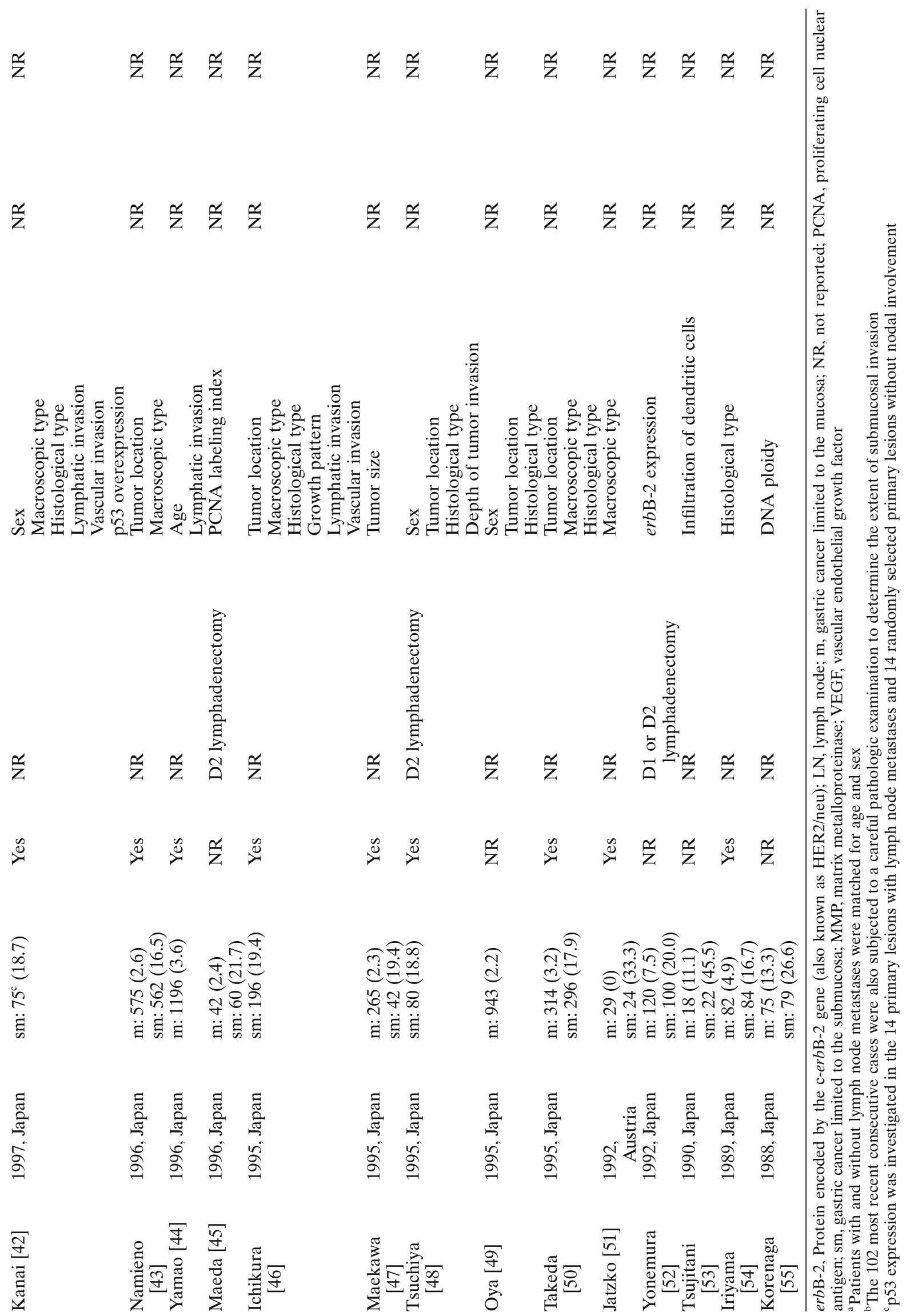




\begin{tabular}{|c|c|c|}
\hline Patient and/or tumor characteristic & References & Pooled odds ratio $(\square)$ or study-specific odds ratio $(O)$ \\
\hline Age; $<57$ years vs. $\geq 57$ years & 44 & \\
\hline $\begin{array}{l}\text { Tumor location; middle part of } \\
\text { the stomach vs. other locations }\end{array}$ & $6,20,23,49,50$ & \\
\hline Tumor size $\cdot$ cut-off $10 \mathrm{~mm}$ & $13,29,30,36,47$ & Upper limit 95\% CI: $\mathbf{1 1 . 6 0}$ \\
\hline - cut-off $20 \mathrm{~mm}$ & $13,29,30,36,47$ & -1 \\
\hline - cut-off $30 \mathrm{~mm}$ & $6,13,30,36,47$ & -1 \\
\hline - cut-off $40 \mathrm{~mm}$ & $29,30,36,47$ & 4 \\
\hline $\begin{array}{l}\text { Macroscopic tumor type; } \\
\text { depressed vs. non-depressed type }\end{array}$ & $\begin{array}{l}6,20,27,30 \\
32,36,43,50\end{array}$ & \\
\hline Tumor ulceration; presence vs. absence & $6,15,20,31,32$ & -1 \\
\hline $\begin{array}{l}\text { Main histological tumor type; } \\
\text { undifferentiated vs. differentiated }\end{array}$ & $15,20,29,49,50,54$ & \\
\hline $\begin{array}{l}\text { Histological tumor subtype according to Lauren } \\
\text { classification; diffuse vs. intestinal/mixed type }\end{array}$ & 20,54 & Upper limit 95\% CI: $\mathbf{1 3 . 2 5}$ \\
\hline $\begin{array}{l}\text { Lymphatic tumor invasion; } \\
\text { presence vs. absence }\end{array}$ & 31,44 & $\begin{array}{l}\text { Pooled odds ratio: } \mathbf{2 2 . 0 7} \\
\text { Upper limit } 95 \% \text { CI: } \mathbf{7 1 . 0 8}\end{array}$ \\
\hline Tumor PCNA labeling index $\geq 25 \%$ vs. $<25 \%$ & 45 & $\begin{array}{l}\text { Odds ratio: } \mathbf{8 1 . 0 0} \\
\text { Upper limit } 95 \% \text { CI: } 2939.74\end{array}$ \\
\hline $\begin{array}{l}\text { MMP-9 expression of the tumor; } \\
\text { presence vs. absence }\end{array}$ & 25 & Upper limit 95\% CI: 14.04 \\
\hline
\end{tabular}

Fig. 1. Forest plot of variables significantly associated with lymph node (LN) metastasis in gastric cancer limited to the mucosa. $P C N A$, Proliferating cell nuclear antigen (an auxiliary protein of the DNA polymerase-delta); $M M P$, matrix metalloproteinase; $C I$, confidence interval

invasion, tumors with a proliferating cell nuclear antigen (PCNA) labeling index of more than $25 \%$, and MMP-9 positive tumors (Fig. 1). Among the studies investigating the variable "tumor ulcerations", more than moderate heterogeneity was identified $\left(\mathrm{I}^{2}=55.8 \%\right)$.

\section{Submucosal cancer}

In patients with submucosal cancer, there was no significant association between age (for various cutoff values), family history of gastric cancer, macroscopic tumor type, tumor ulceration, tumor type according to the Lauren classification, tumor growth pattern, presence of fibrosis near the tumor, tumor stroma, infiltration of dendritic cells, DNA ploidy, p53 overexpression,
GCP-like glandular proliferation of the tumor, vascular endothelial growth factor (VEGF)-D expression, erbB-2 expression, and presence of LN metastasis.

Variables significantly associated with $\mathrm{LN}$ metastasis were: female sex, tumor location in the lower part of the stomach, larger tumor size, undifferentiated tumors, increasing depth of submucosal invasion, lymphatic tumor invasion, vascular tumor invasion, increased submucosal vascularity, tumors with a PCNA labeling index of more than $25 \%$, tumors with a gastric mucin phenotype, and VEGF-C-positive tumors (Fig. 2). Among the studies investigating the variables "main histological tumor type (differentiated vs undifferentiated tumors)" and "vascular tumor invasion", more than moderate heterogeneity was identified (with an $\mathrm{I}^{2}$ of $82.5 \%$ and $51.3 \%$, respectively). 


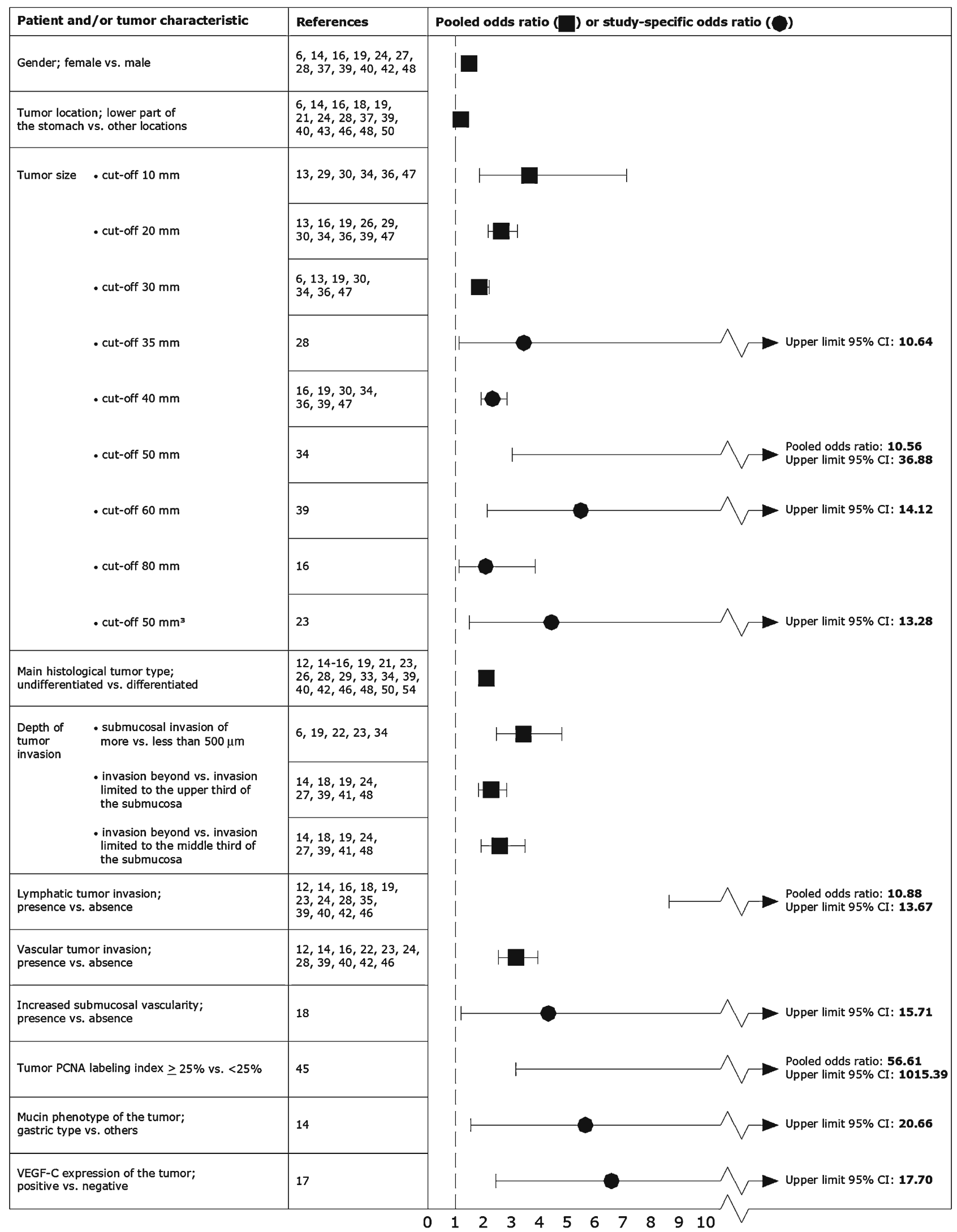

Fig. 2. Forest plot of variables significantly associated with $L N$ metastasis in gastric cancer limited to the submucosa. $P C N A$, Proliferating cell nuclear antigen (an auxiliary protein of the DNA polymerase-delta); $V E G F$, vascular endothelial growth factor 


\section{Discussion}

The overall risk of LN metastasis in mucosal gastric cancer is only about $3.2 \%$ (see Results). In submucosal cancer, the risk of LN metastasis is approximately $19.2 \%$ (see Results). To date, no imaging modality has been proven to be consistently accurate in assessing LN metastasis in EGC. Endoscopic ultrasonography (EUS) mainly depends on LN echogenicity, morphology, and size as criteria to define malignancy. Reported sensitivities and specificities of EUS to detect LN metastases in gastroesophageal carcinomas vary widely, between $59.5 \%$ and $97.2 \%$, and between $40.0 \%$ and $100 \%$ [56]. Computed tomography (CT), another anatomical imaging modality, mainly uses LN size as a criterion to define malignancy. Using a 64-section multidetectorrow CT scanner, a recent study found a sensitivity and specificity of $84.2 \%$ (95\% CI, 62.4-94.5) and $84.0 \%$ (95\% CI, 65.4-93.6) [57]. Still, differentiating between benign and metastatic LNs may be unreliable when LN size is used as a criterion $[58,59] .{ }^{18} \mathrm{~F}$-fluoro-2-deoxyglucose positron emission tomography, a functional imaging modality based on the increased glycolytic rate of malignant cells [60], has also been shown to be insufficiently accurate in assessing LN status in gastric cancer; although reported specificities vary between $90.0 \%$ and $97.0 \%$, reported sensitivities vary between only $34.0 \%$ and $64.6 \%$ [61-63]. The accuracy of other functional imaging modalities, including magnetic resonance imaging (MRI) with ultrasmall particles of superparamagnetic iron oxide [64] and diffusion-weighted MRI [65], still has to be investigated in large patient cohorts. Laparoscopic sentinel node (SN) biopsy is another promising tool to more accurately determine nodal status in EGC patients. The SN concept is based on the premise that tumor cells will preferentially metastasize to the first draining $\mathrm{LN}$ in the regional lymphatics, the SN. After identifying the SN (by use of a radionucleotide tracer and/or dye), and laparoscopic biopsy, LN metastasis is confirmed or ruled out by histological examination. A disadvantage of laparoscopic SN biopsy, however, is its invasiveness. Although studies on laparoscopic SN biopsy have shown its potential [66-69], various technical and material limitations still have to be overcome. Also, the reliability of laparoscopic SN biopsy has yet to be determined by multicenter prospective clinical trials [70].

Because currently available imaging modalities fail to accurately determine nodal status, nodal status in EGC is still predicted by means of the presence or absence of certain tumor characteristics. According to the treatment guidelines of the Japanese Gastric Cancer Association (JGCA), EMR without lymphadenectomy is indicated in differentiated mucosal cancers less than $20 \mathrm{~mm}$ in size, and subtotal gastrectomy with D1 lymph- adenectomy plus resection of the no. $7(+8 a)$ LNs is indicated in undifferentiated mucosal cancers, differentiated mucosal cancers $20 \mathrm{~mm}$ or more in size, and differentiated submucosal cancers $15 \mathrm{~mm}$ or less in size. For other submucosal cancers, the JGCA treatment guidelines indicate subtotal gastrectomy with D1 lymphadenectomy plus resection of the no. 7, 8a, and 9 LNs [5]. These criteria, however, may be too strict and can lead to unnecessary surgery $[3,6]$. Gotoda et al. [6] proposed expanded criteria for the endoscopic treatment of EGC; using the variables "histological tumor type", "lymphatic-vascular involvement", "ulcer findings", and "tumor size", they defined (additional) groups of patients with EGC who may also have been eligible for endoscopic tumor resection. However, for patients who did not belong to one of these groups, the risk of LN metastasis was not reported [6]. Furthermore, there may be more predictive variables allowing an even better risk assessment in the individual EGC patient.

The results of the present systematic review and meta-analysis show that variables significantly associated with LN metastasis in mucosal cancer are: age younger than 57 years, tumor location in the middle part of the stomach, larger tumor size, macroscopically depressed tumor type, tumor ulcerations, undifferentiated tumors, diffuse tumor type according to the Lauren classification [9], lymphatic tumor invasion, tumors with a PCNA labeling index of more than 25\%, and MMP9-positive tumors (Fig. 1). Patients with tumor ulcerations also had a significantly higher risk of LN metastasis. However, more than moderate heterogeneity was identified among the studies investigating this variable. An explanation for this heterogeneity may be the interobserver variability between studies for the assessment of tumor ulcerations. Also, none of the studies investigating this variable mentioned whether assessment of this variable was done blinded to $\mathrm{LN}$ status. Variables significantly associated with LN metastasis in submucosal cancer are: female sex, tumor location in the lower part of the stomach, larger tumor size, undifferentiated tumors, increasing depth of submucosal invasion, lymphatic tumor invasion, vascular tumor invasion, increased submucosal vascularity, tumors with a PCNA labeling index of more than $25 \%$, tumors with a gastric mucin phenotype, and VEGF-C-positive tumors (Fig. 2). It should be noted that more than moderate heterogeneity was identified among the studies investigating the variables "main histological tumor type (differentiated vs undifferentiated tumors)" and "vascular tumor invasion". This may be explained by interobserver variability between studies and the assessment of these variables unblinded to LN status. Another limitation is that certain variables, such as "PNCA labeling index", "MMP-9 expression", "mucin phenotype", and "VEGF-C expression", were investigated 
only by single and relatively small-sized studies. This is also expressed by their wide confidence intervals (see Figs. 1 and 2).

Lymphatic tumor invasion was the strongest univariate predictor for LN metastasis in both mucosal and submucosal gastric cancer. This is not surprising, as the lymphatics are the direct pathway to the LNs. However, the JGCA treatment guidelines are not based on the presence or absence of lymphatic tumor invasion [5]. JGCA treatment guidelines use a tumor size of $20 \mathrm{~mm}$ as the cutoff in differentiated mucosal cancer to decide for either endoscopic resection or modified gastrectomy. This was based on the assumption that differentiated mucosal cancer of $20 \mathrm{~mm}$ or less has no LN metastasis, and also because $20 \mathrm{~mm}$ was the technical upper limit of en-bloc resection at the time the guidelines were composed [5]. However, newer endoscopic resection techniques allow the en-bloc resection of larger lesions [2]. Future studies should assess whether there is a significant difference in LN metastasis risk between different tumor size cutoff values, after applying multivariate analysis to adjust for other variables. Notably, although a large size of carcinoma can contribute to contact between carcinoma cells and a lymphoid vessel, specific enzymes are necessary for the degradation of the vessel wall and for the invasion of the lymphoid vessel. MMPs are considered to be important for the facilitation of tumor invasion and spread [71]. Indeed, MMP-9 was significantly associated with LN metastasis in mucosal cancer [31]. To our knowledge, the relation between MMPs and LN metastasis in submucosal cancer still has to be investigated. It should also be further explored which MMPs (or combinations of MMPs) are most predictive for $\mathrm{LN}$ metastasis.

Despite the findings of our univariate analysis, confounding of variables may be present. For instance, lymphatic tumor invasion, vascular tumor invasion, and VEGF-C are all significantly associated with LN metastasis, but tumor angiogenesis and lymphangiogenesis (which may both promote lymphatic and vascular tumor invasion) are also related to VEGF-C. In addition, it is also very likely that the variables "main tumor histology (differentiated vs undifferentiated type)" and "tumor histology according to the Lauren classification" [9] are overlapping. Thus, future studies are needed to assess which variables are significant predictors of LN metastasis after multivariate analysis. These variables can then be used to develop a model that can accurately predict the risk of $\mathrm{LN}$ metastasis in an individual patient.

Of note, in 1989 Kampschoer et al. [72] had already developed a computer program to predict the probability of LN metastasis, based on certain histopathological features of the primary tumor. However, the point at which LN dissection should be done, based on this program, is not clear [73]. In addition, the computer program is based only on the preoperative variables "sex", "age", "tumor location", "macroscopic type", "tumor size", "depth of tumor invasion", and "histological type" [72]. For instance, both lymphatic and vascular invasion, which proved to be strongly associated with LN metastasis by our analysis, were not used by the program.

It should also be noted that, in the study of Kampschoer et al. [72], depth of tumor invasion and histological type were assessed by means of double-contrast $\mathrm{X}$-rays and histological analysis of biopsied tissue. Endoscopic ultrasound was performed if results were inconclusive. Kampschoer et al. [72] stated that the preoperative diagnosis and classification were correlated with the surgical findings in $96.5 \%$ of cases, and that the extent of invasion could be accurately assessed because of the expertise of the radiologists. In actual practice, however, the accuracy of histopathologic grading using forceps biopsy specimens is only approximately $82.5 \%$ when compared to the final histological typing based on the predominant histology of the resected tumor [74]. In addition, it is still unclear whether EUS, the current first-choice imaging modality in T-staging, can accurately differentiate between mucosal and deeper gastric cancer [75]. When biopsy specimens do not reflect the predominant histopathology of the entire tumor and/or the depth of invasion as assessed by EUS is greater than the actual depth, a decision may be made to perform surgery unnecessarily. Endoscopic resection, however, has the ability to provide complete histopathological staging (and assessing all variables) without precluding future surgery [3,76]. Therefore, in all patients who are suspected of having EGC or in cases in which the biopsy-based histopathologic typing or EUSdetermined depth of invasion is thought to be unreliable, endoscopic resection should be performed as the first step. Then, after evaluating the resected specimen, one can weigh the risks of LN metastasis against the risk of surgery.

Although the majority of the studies included in the present systematic review and meta-analysis were performed in Japan, it is likely that the results of this meta-analysis are generalizable, because the clinicopathological features of gastric cancer in Japan and Western countries do not seem to differ [77]. Of the included studies, $16 \%$ performed D2 or more extensive lymphadenectomy in all patients. D1 lymphadenectomy includes the removal of level 1 LNs only, whereas D2 lymphadenectomy adds the removal of level 2 LNs [8]. Although metastasis to level $2 \mathrm{LNs}$ is rare, occurring in only $0.4 \%$ of patients with mucosal cancer and in $4.9 \%$ of patients with submucosal cancer [78], some skip metastases may have been missed in patients who 
underwent less than D2 lymphadenectomy. Another limitation of the included studies is that all but one did not assess patient and/or tumor characteristics blinded to LN status, and vice versa, which may have introduced bias.

In conclusion, the present systematic review and meta-analysis identified several variables that are associated with LN metastasis in EGC. These variables should be included in future research, in order to assess which of these variables remain as significant predictors of LN metastasis.

Acknowledgments We acknowledge Professor R.G.H. Beets-Tan for her expert review of the manuscript.

\section{References}

1. Rubin E, Palazzo JP. The gastrointestinal tract. In: Rubin E, Gorstein F, Rubin F, Schwarting R, Strayer D, editors. Rubin's pathology. Clinicopathologic foundations of medicine. 4th ed. Philadelphia: Lippincott Williams \& Wilkins; 2005. p. 660-739.

2. Siewert JR. Gastric cancer: the dispute between East and West. Gastric Cancer 2005;8:59-61.

3. Soetikno R, Kaltenbach T, Yeh R, Gotoda T. Endoscopic mucosal resection for early cancers of the upper gastrointestinal tract. J Clin Oncol 2005;23:4490-8.

4. Brennan MF. Current status of surgery for gastric cancer: a review. Gastric Cancer 2005;8:64-70.

5. Nakajima T. Gastric cancer treatment guidelines in Japan. Gastric Cancer 2002;5:1-5.

6. Gotoda T, Yanagisawa A, Sasako M, Ono H, Nakanishi Y, Shimoda T, et al. Incidence of lymph node metastasis from early gastric cancer: estimation with a large number of cases at two large centers. Gastric Cancer 2000;3:219-25.

7. Yonemura Y, Endo Y, Hayashi I, Kawamura T, Yun HY, Bandou E. Proliferative activity of micrometastases in the lymph nodes of patients with gastric cancer. Br J Surg 2007;94:731-6.

8. Japanese Gastric Cancer Association. Japanese classification of gastric carcinoma - 2nd English edition. Gastric Cancer 1998;1:10-24.

9. Lauren $\mathrm{P}$. The two histological main types of gastric carcinoma: diffuse and so-called intestinal-type carcinoma. Acta Pathol Microbiol Imunol Scand 1965;64:31-49.

10. Mantel N, Haenszel W. Statistical aspects of the analysis of data from retrospective studies of disease. J Natl Cancer Inst 1959; 22:719-48.

11. Higgins JP, Thompson SG, Deeks JJ, Altman DG. Measuring inconsistency in meta-analyses. BMJ 2003;327:557-60.

12. Okabayashi T, Kobayashi M, Sugimoto T, Okamoto K, Hokimoto N, Araki K. Clinicopathological investigation of early gastric carcinoma; is less invasive surgery right for early gastric carcinoma? Hepatogastroenterology 2007;54:609-12.

13. Lo SS, Wu CW, Chen JH, Li AF, Hsieh MC, Shen KH, et al. Surgical results of early gastric cancer and proposing a treatment strategy. Ann Surg Oncol 2007;14:340-7.

14. Nakamoto J, Torisu R, Aoki R, Kimura Y, Yasuda M, Shiota K, et al. Clinicopathological evaluation of biological behavior of submucosal invasive gastric carcinomas: relationship among lymph node metastasis, mucin phenotype and proliferative activity. J Med Invest 2007;54:99-108.

15. Ishikawa $\mathrm{S}$, Togashi $\mathrm{A}$, Inoue $\mathrm{M}$, Honda $\mathrm{S}$, Nozawa F, Toyama $\mathrm{E}$, et al. Indications for EMR/ESD in cases of early gastric cancer: relationship between histological type, depth of wall invasion, and lymph node metastasis. Gastric Cancer 2007;10:35-8.

16. Kunisaki C, Akiyama H, Nomura M, Matsuda G, Otsuka Y, Ono HA, et al. Lymph node status in patients with submucosal gastric cancer. Ann Surg Oncol 2006;13:1364-71.

17. Onogawa S, Kitadai Y, Amioka T, Kodama M, Cho S, Kuroda T, et al. Expression of vascular endothelial growth factor (VEGF)-C and VEGF-D in early gastric carcinoma: correlation with clinicopathological parameters. Cancer Lett 2005;226:85-90.

18. Son HJ, Song SY, Kim S, Noh JH, Sohn TS, Kim DS, et al. Characteristics of submucosal gastric carcinoma with lymph node metastatic disease. Histopathology 2005;46:158-65.

19. Park DJ, Lee HK, Lee HJ, Lee HS, Kim WH, Yang HK, et al. Lymph node metastasis in early gastric cancer with submucosal invasion: feasibility of minimally invasive surgery. World J Gastroenterol 2004;10:3549-52.

20. Song SY, Park S, Kim S, Son HJ, Rhee JC. Characteristics of intramucosal gastric carcinoma with lymph node metastatic disease. Histopathology 2004;44:437-44.

21. Yoshikawa T, Tsuburaya A, Kobayashi O, Sairenji M, Motohashi $\mathrm{H}$, Noguchi Y. Indications of limited surgery for gastric cancer with submucosal invasion-analysis of 715 cases with special reference to site of the tumor and level 2 lymph nodes. Hepatogastroenterology 2003;50:1727-30.

22. Higashi H, Natsugoe S, Ishigami S, Uenosono Y, Matsumoto M, Nakajo A, et al. Distribution of lymph node metastasis including micrometastasis in gastric cancer with submucosal invasion. World J Surg 2003;27:455-9.

23. Matsuzaki H, Kikuchi S, Kakita A. Evaluation of the morphology of submucosal tumor invasion and its volume in early gastric cancer. In Vivo 2003;17:41-4.

24. Shimoyama S, Yasuda H, Mafune K, Kaminishi M. Indications of a minimized scope of lymphadenectomy for submucosal gastric cancer. Ann Surg Oncol 2002;9:625-31.

25. Kabashima A, Yao T, Sugimachi K, Tsuneyoshi M. Relationship between biologic behavior and phenotypic expression in intramucosal gastric carcinomas. Hum Pathol 2002;33:80-6.

26. Takeno S, Noguchi T, Kikuchi R, Sato T, Uchida Y, Yokoyama S. Analysis of early (pT1) gastric cancer with submucosal invasion: surgical management and possibility to schedule less invasive surgery. Ann Surg Oncol 2001;8:605-10.

27. Shimada S, Yagi Y, Shiomori K, Honmyo U, Hayashi N, Matsuo A, et al. Characterization of early gastric cancer and proposal of the optimal therapeutic strategy. Surgery 2001;129:714-9.

28. Yamada H, Nihei Z, Yamashita T, Shirota Y, Ichikawa W, Sugihara $\mathrm{K}$. Is lymphadenectomy needed for all submucosal gastric cancers? Eur J Surg 2001;167:199-203.

29. Seto Y, Shimoyama S, Kitayama J, Mafune K, Kaminishi M, Aikou T, et al. Lymph node metastasis and preoperative diagnosis of depth of invasion in early gastric cancer. Gastric Cancer 2001:4:34-8.

30. Shimoyama S, Seto Y, Yasuda H, Kaminishi M. Wider indications for the local resection of gastric cancer by adjacent lymphadenectomy. J Surg Oncol 2000;75:157-64.

31. Kabashima A, Maehara Y, Kakeji Y, Baba H, Koga T, Sugimachi $\mathrm{K}$. Clinicopathological features and overexpression of matrix metalloproteinases in intramucosal gastric carcinoma with lymph node metastasis. Clin Cancer Res 2000;6:3581-4.

32. Jiang BJ, Sun RX, Lin H, Gao YF. Study on the risk factors of lymphatic metastasis and the indications of less in vasive operations in early gastric cancer. World J Gastroenterol 2000;6: 553-6.

33. Takano Y, Teranishi Y, Terashima S, Motoki R, Kawaguchi T. Lymph node metastasis-related carbohydrate epitopes of gastric cancer with submucosal invasion. Surg Today 2000;30:1073-82.

34. Yasuda K, Shiraishi N, Suematsu T, Yamaguchi K, Adachi Y, Kitano S. Rate of detection of lymph node metastasis is correlated with the depth of submucosal invasion in early stage gastric carcinoma. Cancer 1999;85:2119-23. 
35. Ishigami $S$, Natsugoe $S$, Hokita $S$, Tokushige $M$, Saihara $T$, Watanabe T, et al. Carcinomatous lymphatic invasion in early gastric cancer invading into the submucosa. Ann Surg Oncol 1999; 6286-9.

36. Tsujitani S, Oka S, Saito H, Kondo A, Ikeguchi M, Maeta M, et al. Less invasive surgery for early gastric cancer based on the low probability of lymph node metastasis. Surgery 1999;125: 148-54.

37. Ishigami S, Hokita S, Natsugoe S, Tokushige M, Saihara T, Iwashige $\mathrm{H}$, et al. Carcinomatous infiltration into the submucosa as a predictor of lymph node involvement in early gastric cancer. World J Surg 1998;22:1056-9.

38. Namieno T, Koito K, Higashi T, Shimamura T, Yamashita K, Kondo Y. Tumor recurrence following resection for early gastric carcinoma and its implications for a policy of limited resection. World J Surg 1998;22:869-73.

39. Kurihara N, Kubota T, Otani Y, Ohgami M, Kumai K, Sugiura $\mathrm{H}$, et al. Lymph node metastasis of early gastric cancer with submucosal invasion. Br J Surg 1998;85:835-9.

40. Morita M, Baba H, Fukuda T, Taketomi A, Kohnoe S, Seo Y, et al. Submucosal gastric cancer with lymph node metastasis. J Surg Oncol 1998;68:5-10.

41. Takeshita K, Saeki I, Tani M, Honda T, Saito N, Endo M. Rational lymphadenectomy for early gastric cancer with submucosal invasion: a clinicopathological study. Surg Today 1998;28: $580-6$.

42. Kanai T, Konno H, Maruyama K, Baba M, Tanaka T, Maruo Y, et al. p53 overexpression and proliferative activity do not correlate with lymph node metastasis in early gastric cancer. Eur Surg Res 1997;29:35-41.

43. Namieno T, Koito K, Higashi T, Sato N, Uchino J. General pattern of lymph node metastasis in early gastric carcinoma. World J Surg 1996;20:996-1000.

44. Yamao T, Shirao K, Ono H, Kondo H, Saito D, Yamaguchi H, et al. Risk factors for lymph node metastasis from intramucosal gastric carcinoma. Cancer 1996;77:602-6.

45. Maeda K, Chung YS, Onoda N, Ogawa M, Kato Y, Nitta A, et al. Association of tumor cell proliferation with lymph node metastasis in early gastric cancer. Oncology 1996;53:1-5.

46. Ichikura T, Uefuji K, Tomimatsu S, Okusa Y, Yahara T, Tamakuma S. Surgical strategy for patients with gastric carcinoma with submucosal invasion. A multivariate analysis. Cancer 1995;76: 935-40.

47. Maekawa S, Takeo S, Ikejiri K, Anai H, Saku M. Clinicopathological features of lymph node metastasis in early gastric cancer. Int Surg 1995;80:200-3.

48. Tsuchiya A, Kikuchi Y, Ando Y, Yoshida T, Abe R. Lymph node metastases in gastric cancer invading the submucosal layer. Eur J Surg Oncol 1995;21:248-50.

49. Oya M, Yao T, Nagai E, Tsuneyoshi M. Metastasizing intramucosal gastric carcinomas. Well differentiated type and proliferative activity using proliferative cell nuclear antigen and Ki-67. Cancer 1995;75:926-35.

50. Takeda J, Toyonaga A, Koufuji K, Kodama I, Aoyagi K, Ohta J, et al. Resected early gastric cancer-clinicopathological studies on 610 cases. Kurume Med J 1995;42:87-94.

51. Jatzko G, Lisborg PH, Klimpfinger M. Extended lymphadenectomy against early gastric cancer. Jpn J Clin Oncol 1992;22: 26-9.

52. Yonemura Y, Ninomiya I, Ohoyama S, Fushida S, Kimura H, Tsugawa K, et al. Correlation of c-erbB-2 protein expression and lymph node status in early gastric cancer. Oncology 1992;49: 363-7.

53. Tsujitani S, Kakeji Y, Watanabe A, Kohnoe S, Maehara Y, Sugimachi K. Infiltration of dendritic cells in relation to tumor invasion and lymph node metastasis in human gastric cancer. Cancer 1990;66:2012-6.

54. Iriyama K, Asakawa T, Koike H, Nishiwaki H, Suzuki H. Is extensive lymphadenectomy necessary for surgical treatment of intramucosal carcinoma of the stomach? Arch Surg 1989;124: 309-11.

55. Korenaga D, Okamura T, Saito A, Baba H, Sugimachi K. DNA ploidy is closely linked to tumor invasion, lymph node metastasis, and prognosis in clinical gastric cancer. Cancer 1988;62:30913 .

56. Kelly S, Harris KM, Berry E, Hutton J, Roderick P, Cullingworth $\mathrm{J}$, et al. A systematic review of the staging performance of endoscopic ultrasound in gastro-oesophageal carcinoma. Gut 2001;49: 534-9.

57. Yang DM, Kim HC, Jin W, Ryu CW, Kang JH, Park CH, et al. 64 multidetector-row computed tomography for preoperative evaluation of gastric cancer: histological correlation. J Comput Assist Tomogr 2007;31:98-103.

58. Monig SP, Zirbes TK, Schroder W, Baldus SE, Lindemann DG, Dienes HP, et al. Staging of gastric cancer: correlation of lymph node size and metastatic infiltration. AJR Am J Roentgenol 1999;173:365-7.

59. Nakamura K, Morisaki T, Noshiro H, Torata N, Kinukawa N, Tanaka M. Morphometric analysis of regional lymph nodes with and without metastasis from early gastric carcinoma. Cancer 2000;88:2438-42.

60. Rohren EM, Turkington TG, Coleman RE. Clinical applications of PET in oncology. Radiology 2004;231:305-32.

61. Mukai K, Ishida $\mathrm{Y}$, Okajima $\mathrm{K}$, Isozaki $\mathrm{H}$, Morimoto $\mathrm{T}$, Nishiyama S. Usefulness of preoperative FDG-PET for detection of gastric cancer. Gastric Cancer 2006;9:192-6.

62. Yun M, Lim JS, Noh SH, Hyung WJ, Cheong JH, Bong JK, et al. Lymph node staging of gastric cancer using (18) F-FDG PET: a comparison study with CT. J Nucl Med 2005;46:1582-8.

63. Tian J, Chen L, Wei B, Shao M, Ding Y, Yin D, et al. The value of vesicant $18 \mathrm{~F}$-fluorodeoxyglucose positron emission tomography (18F-FDG PET) in gastric malignancies. Nucl Med Commun 2004;25:825-31.

64. Tatsumi Y, Tanigawa N, Nishimura H, Nomura E, Mabuchi H, Matsuki M, et al. Preoperative diagnosis of lymph node metastases in gastric cancer by magnetic resonance imaging with ferumoxtran-10. Gastric Cancer 2006;9:120-8.

65. Shinya S, Sasaki T, Nakagawa Y, Guiquing Z, Yamamoto F, Yamashita Y. The usefulness of diffusion-weighted imaging (DWI) for the detection of gastric cancer. Hepatogastroenterology 2007;54:1378-81.

66. Hiratsuka M, Miyashiro I, Ishikawa O, Furukawa H, Motomura $\mathrm{K}$, Ohigashi $\mathrm{H}$, et al. Application of sentinel node biopsy to gastric cancer surgery. Surgery 2001;129:335-40.

67. Tonouchi H, Mohri Y, Tanaka K, Konishi N, Ohmori Y, Kobayashi M, et al. Lymphatic mapping and sentinel node biopsy during laparoscopic gastrectomy for early cancer. Dig Surg 2003;20:421-7.

68. Song X, Wang L, Chen W, Pan T, Zhu H, Xu J, et al. Lymphatic mapping and sentinel node biopsy in gastric cancer. Am J Surg 2004;187:270-3.

69. Mura G, Vagliasindi A, Framarini M, Mazza P, Solfrini G, Verdecchia GM. The sentinel node biopsy in early gastric cancer: a preliminary study. Langenbecks Arch Surg 2006;391:113-7.

70. Kitagawa Y, Fujii H, Kumai K, Kubota T, Otani Y, Saikawa Y, et al. Recent advances in sentinel node navigation for gastric cancer: a paradigm shift of surgical management. J Surg Oncol 2005;90:147-51.

71. Parsons SL, Watson SA, Brown PD, Collins HM, Steele RJ. Matrix metalloproteinases. Br J Surg 1997;84:160-6.

72. Kampschoer GH, Maruyama K, van de Velde CJ, Sasako M, Kinoshita T, Okabayashi K. Computer analysis in making preoperative decisions: a rational approach to lymph node dissection in gastric cancer patients. Br J Surg 1989;76:905-8.

73. Hohenberger P, Gretschel S. Gastric cancer. Lancet 2003;362: 305-15.

74. Matsubara Y, Yanai H, Ishiguro K, Ryozawa S, Okazaki Y, Matsui N, et al. Clinical interpretation of the histological typing 
of gastric cancer using endoscopic forceps biopsy. Hepatogastroenterology 2004;51:285-8.

75. Kwee RM, Kwee TC. The accuracy of endoscopic ultrasonography in differentiating mucosal from deeper gastric cancer. Am J Gastroenterol 2008;103:1801-9.

76. Gotoda T. Endoscopic resection of early gastric cancer. Gastric Cancer 2007;10:1-11.
77. Everett SM, Axon AT. Early gastric cancer in Europe. Gut 1997;41:142-50.

78. Yoshikawa T, Tsuburaya A, Kobayashi O, Sairenji M, Motohashi $\mathrm{H}$, Noguchi Y. Is D2 lymph node dissection necessary for early gastric cancer? Ann Surg Oncol 2002;9:401-5. 